\title{
Optimization of Droplet Routing for an $n$-Plex Bioassay on a Digital Microfluidic Lab-on-Chip*
}

\author{
Yang Zhao \\ Duke University \\ Durham, NC 27708 \\ Email: yz61@ee.duke.edu
}

\author{
Ryan Sturmer \\ Advanced Liquid Logic, Inc. \\ Research Triangle Park, NC 27560 \\ Email: rsturmer@Liquid-Logic.com
}

\author{
Krishnendu Chakrabarty \\ Duke University \\ Durham, NC 27708 \\ Email: krish@ee.duke.edu
}

\author{
Vamsee K. Pamula \\ Advanced Liquid Logic, Inc. \\ Research Triangle Park, NC 27560 \\ Email: VPamula@Liquid-Logic.com
}

\begin{abstract}
The multiplexed in-vitro measurement of glucose and other metabolites in human physiological fluids is of great importance for the clinical diagnosis of metabolic disorders. We present a design technique for optimizing an $n$-plex bioassay on an electrowetting-based digital microfluidic lab-on-chip. The $n$ product droplets of the $n$-plex bioassay are detected using a photomultiplier tube located at the transport bus of the lab-on-chip. We optimize the routing of the droplets and prove that this approach minimizes the assay completion time. The effectiveness of the proposed droplet-routing schedule is shown via an on-chip experiment for a 3-plex assay. We compare the completion time of the proposed routing schedule to an unoptimized baseline schedule for the $n$-plex assay.
\end{abstract}

\section{INTRODUCTION}

The in-vitro measurement of glucose and other electrolytes and gases in human physiological fluids is of great importance for clinical diagnosis. For instance, the change in regular metabolic parameters in the patient's blood can signal organ damage or dysfunction prior to observable microscopic cellular damages or other symptoms. A portable, inexpensive lab-on-chip can be used for carrying out multiplexed bioassays for rapid and point-of-care diagnosis of such disorders.

Digital microfluidic lab-on-chip is an emerging technology that aims to miniaturize and integrate fluid-handling onto a chip [1], [4]. Compared to traditional bench-top procedures, a microfluidic lab-ona-chip offers the advantages of low sample and reagent consumption, less likelihood of error due to minimal human intervention, high throughput, and high sensitivity. Recent years have seen a steady increase in the level of integration and system complexity of digital microfluidic chips [1]. These advances in technology serve as a powerful driver for research on computer-aided design (CAD) tools for the design of such chips [10]-[14].

The feasibility of performing enzymatic assays [8], DNA amplification assays [6], DNA sequencing [1], and immunoassays [9] have been successfully demonstrated on a digital microfluidic system, wherein the droplets were manipulated electric fields. Furthermore, these assays can be integrated for multiplexed in-vitro diagnostics on different human physiological fluids [7]. Recently, cell-based applications have been demonstrated using digital microfluidics [4].

The $n$-plex bioassay is a typical example of multiplexed and concurrent bioassays. In an $n$-plex assay, a sample is analyzed for $n$ different analytes. Sample droplets are mixed with $n$ different reagents, the mixed products undergo incubation, and the incubated product droplets are routed to a detection site. The signal transduction for quantifying the $n$ droplets can be based on an optical signal from each droplet. An optical detector such as a photodiode or a photomultiplier tube (PMT) [5] can be used to analyze the product droplets of the $n$-plex bioassay. However, the detection process is time-consuming since there are $n$ product droplets to be detected for the $n$-plex bioassay. Moreover, undesirable waiting times become

* This work was supported in part by the National Institute of General Medical Sciences of the National Institute of Health (grant \# R44GM072155) and the National Science Foundation (grant \# CCF-0541055). inevitable if the $n$ product droplets are not routed carefully. Therefore, there is a need to optimize the droplet-routing schedule to minimize the completion time for detection.

In this paper, we propose an optimal droplet-routing schedule for an $n$-plex bioassay and a prototype digital microfluidic lab-onchip. We formally prove that this schedule indeed minimizes the completion time. A representative 3-plex assay is performed on a fabricated digital microfluidic platform to show the effectiveness of the proposed schedule. We compare the completion time of the proposed schedule to an unoptimized baseline schedule for the $n$-plex assay.

\section{Digital Microfluidic Platform}

A digital microfluidic biochip utilizes the phenomenon of electrowetting to manipulate and move nanoliter droplets containing biological samples on a two-dimensional electrode array [1]. A unit cell in the array includes a pair of electrodes that acts as two parallel plates. The bottom plate contains a patterned array of individually controlled electrodes, and the top plate is coated with a continuous ground electrode. A droplet rests on a hydrophobic surface over an electrode. Droplets are moved by applying a control voltage to a unit cell adjacent to the droplet and, at the same time, deactivating the one just under the droplet. This electronic method of wettability control creates interfacial tension gradients that move the droplets to the charged electrode.

Fluid-handling operations such as droplet merging, splitting, mixing, and dispensing can be executed in a similar manner. The digital microfluidic platform offers the additional advantage of flexibility, referred to as reconfigurability, since fluidic operations can be performed anywhere on the array. Droplet routes and operation scheduling result are programmed into a microcontroller that drives electrodes in the array.

\section{Prototype Chip Layout And Pin Assignment For AN $n$-PLEX BIOASSAY}

The prototype chip layout for the $n$-plex assay is shown in Fig. 1. The chip consists of three reservoirs. The reservoir called "wash" is used to store the washing droplets, which are used to clean the pathway of electrodes on which product droplets are transported. The reservoir called "substrate" is used to store the substrate droplets, which are mixed with the product droplets, which contain the enzymes, to catalyse the substrate to produce an enzymatic product for optical detection. The reservoir called "waste" is used to store the residual droplets after the detection process is completed. There are $n$ "reactors" at the right of the chip layout. The reactors can be used to implement microfluidic operations such as transporting, mixing, splitting and storing. A one-dimensional transport bus is placed to connect the reactors and the reservoirs. Optical detection is performed at the "detection spot".

The electrodes are connected to control pins for electrical activation. Early $\mathrm{CAD}$ research on digital microfluidic chips assumed a direct-addressing scheme for the control of electrodes [2]. Each 


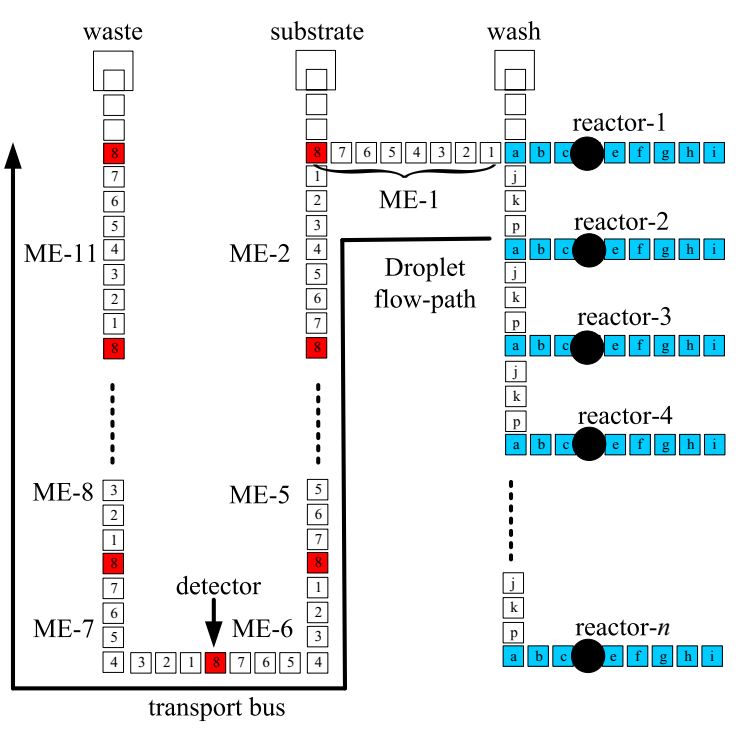

Fig. 1. Prototype chip layout for the $n$-plex assay.

electrode is assumed to be connected to a dedicated control pin; it can therefore be activated independently. This method allows the maximum freedom of droplet manipulation, but it necessitates an impractically excessive number of control pins. Electrode addressing methods that allow the control of digital microfluidic arrays with a small number of pins are therefore more practical [8], [11], [13], [14]. In the method proposed in [8], the number of control pins is minimized by using a multi-phase bus for the fluidic pathways. Every $n$th electrode in an $n$-phase bus is electrically connected, where $n$ is small number (typically $n=4$ ). The method presented in [14] uses array partitioning and careful pin assignment to reduce the number of control pins. A "cross referencing" design is proposed in [13], where an electrode is connected to two pins, corresponding to a row and a column, respectively. In [11], a basic integer linear programming (ILP) formulation is proposed to optimally solve the droplet routing problem for cross-referencing lab-on-chips.

The prototype chip layout for the $n$-plex assay is based on a "busbased" pin-constrained design that utilizes limited number of input pins to control the electrodes. The pin assignment is indicated in Fig. 1. The electrodes in one reactor are controlled by independent control pins $(a, b, \ldots, i)$, while the electrodes in the same column of the $n$ reactors share the same control pin. The transport bus consists of eleven meta-electrodes that are indicated as "ME-1", "ME2", ..., "ME-11". Each meta-electrode is a group of eight adjacent electrodes in the one-dimensional transport bus. The eight electrodes in a meta-electrode are independently controlled by input pins, while the electrodes in the same position of the eleven meta-electrodes share the same control pin. One macro-cycle is defined as the time needed for one droplet to traverse a meta-electrode. Therefore, one macro-cycle equal eight clock cycles.

\section{DRoplet-Routing Optimization}

The final products of an $n$ assay, i.e., $n$ unit-volume product droplets, are located in the same column of reactor- 1 , reactor- $2, \ldots$, reactor- $n$, as shown in Fig. 1. We next have to transport each product droplet to the detector site and place it there for five clock cycles for detection as per the assay protocol [7]. Next the product droplet must be moved to the waste reservoir. Each product droplet needs to mix with a droplet dispensed from the substrate reservoir before reaching the detector. In order to clean up the droplet pathways after each product droplet, three wash droplets must be routed serially to

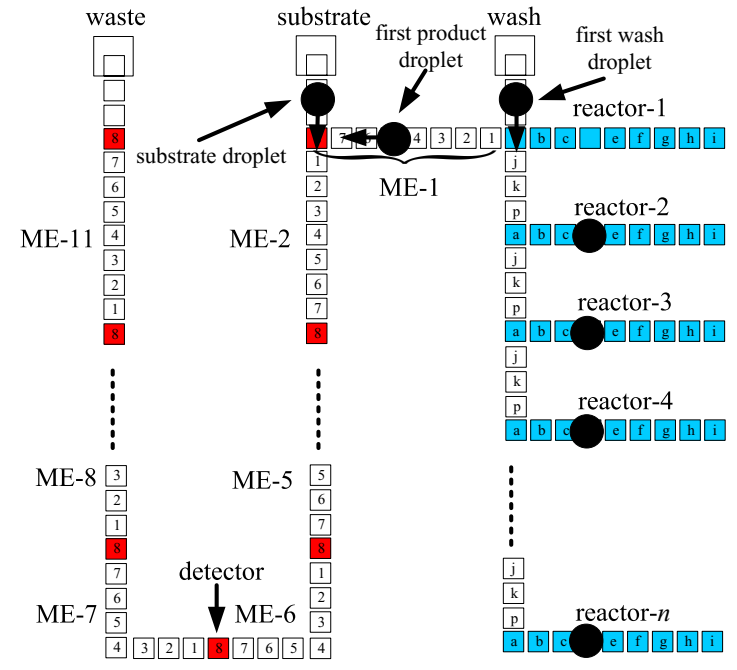

Fig. 2. Step 1 of the detection stage for the 1st product droplet of the $n$-plex assay.

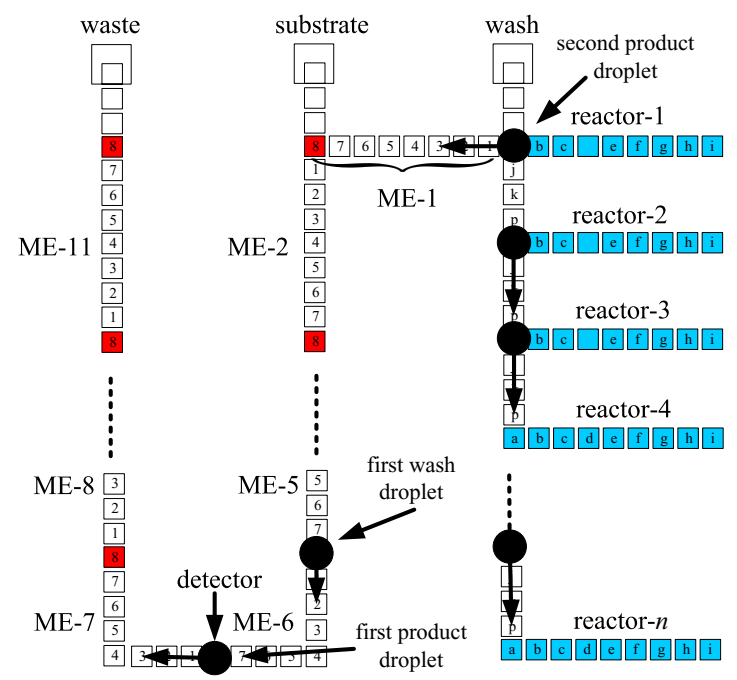

Fig. 3. The beginning of the detection process for the 2 nd product droplet of the $n$-plex assay.

traverse the transport bus until they reach the waste reservoir after each product droplet. The detection process requires that the interval between two droplets in the transport bus is one meta-electrode. Additionally, it is required that the next product droplet cannot move into the transport bus until the previous product droplet leaves the detector. Given the prototype chip layout and the pin assignment, we optimize the schedule for the detection process to minimize the completion time for the detection of $n$ product droplets.

To minimize the completion time for the detection step of the $n$ plex assay, we synchronize multiple microfluidic operations under the pin constraints of the prototype chip layout. Two operations can be synchronized, i.e., executed concurrently, only if there is no conflict in the activation of the pins for the two corresponding actuation sequences.

The 1st product droplet starts moving from the leftmost electrode of reactor- 1 to Electrode 8 of ME- 1 at the beginning of the 1 st macrocycle. At the same time, one substrate droplet is dispensed from the substrate reservoir, while the 1st wash droplet is also dispensed from wash reservoir, as shown in Fig. 2. The product droplet mixes with the substrate droplet during the 1st macro-cycle. The above three microfluidic operations are synchronized to minimize the completion 
TABLE I

THE OPTIMAL DROPLET-ROUTING SCHEDULE FOR THE 3-PLEX ASSAY.

\begin{tabular}{|c|c|c|c|c|c|c|c|c|c|c|c|c|c|}
\hline \multirow{2}{*}{$\begin{array}{c}\text { Macro } \\
\text { cycle }\end{array}$} & \multirow{2}{*}{$\begin{array}{c}\text { Substrate } \\
\text { droplet }\end{array}$} & \multirow{2}{*}{$\begin{array}{c}\text { Wash } \\
\text { operation }\end{array}$} & \multicolumn{11}{|c|}{ Droplet on the different meta-electrodes } \\
\hline & & & ME-1 & ME-2 & ME-3 & ME-4 & ME-5 & ME-6 & ME-7 & ME-8 & ME-9 & ME-10 & ME-11 \\
\hline 1 & $S B_{1}$ & $W_{1,1}$ & $D_{1}$ & & & & & & & & & & \\
\hline 2 & & $W_{1,2}$ & $W_{1,1}$ & $D_{1}$ & & & & & & & & & \\
\hline 3 & & $W_{1,3}$ & $W_{1,2}$ & $W_{1,1}$ & $D_{1}$ & & & & & & & & \\
\hline 4 & & & $W_{1,3}$ & $W_{1,2}$ & $W_{1,1}$ & $D_{1}$ & & & & & & & \\
\hline 5 & & & & $W_{1,3}$ & $W_{1,2}$ & $W_{1,1}$ & $D_{1}$ & & & & & & \\
\hline 6 & & & & & $W_{1,3}$ & $W_{1,2}$ & $W_{1,1}$ & $D_{1}$ & & & & & \\
\hline 7 & $S B_{2}$ & $W_{2,1}$ & $D_{2}$ & & & $W_{1,3}$ & $W_{1,2}$ & $W_{1,1}$ & $D_{1}$ & & & & \\
\hline 8 & & $W_{2,2}$ & $W_{2,1}$ & $D_{2}$ & & & $W_{1,3}$ & $W_{1,2}$ & $W_{1,1}$ & $D_{1}$ & & & \\
\hline 9 & & $W_{2,3}$ & $W_{2,2}$ & $W_{2,1}$ & $D_{2}$ & & & $W_{1,3}$ & $W_{1,2}$ & $W_{1,1}$ & $D_{1}$ & & \\
\hline 10 & & & $W_{2,3}$ & $W_{2,2}$ & $W_{2,1}$ & $D_{2}$ & & & $W_{1,3}$ & $W_{1,2}$ & $W_{1,1}$ & $D_{1}$ & \\
\hline 11 & & & & $W_{2,3}$ & $W_{2,2}$ & $W_{2,1}$ & $D_{2}$ & & & $W_{1,3}$ & $W_{1,2}$ & $W_{1,1}$ & $D_{1}$ \\
\hline 12 & & & & & $W_{2,3}$ & $W_{2,2}$ & $W_{2,1}$ & $D_{2}$ & & & $W_{1,3}$ & $W_{1,2}$ & $W_{1,1}$ \\
\hline 13 & $S B_{3}$ & $W_{3,1}$ & $D_{3}$ & & & $W_{2,3}$ & $W_{2,2}$ & $W_{2,1}$ & $D_{2}$ & & & $W_{1,3}$ & $W_{1,2}$ \\
\hline 14 & & $W_{3,2}$ & $W_{3,1}$ & $D_{3}$ & & & $W_{2,3}$ & $W_{2,2}$ & $W_{2,1}$ & $D_{2}$ & & & $W_{1,3}$ \\
\hline 15 & & $W_{3,3}$ & $W_{3,2}$ & $W_{3,1}$ & $D_{3}$ & & & $W_{2,3}$ & $W_{2,2}$ & $W_{2,1}$ & $D_{2}$ & & \\
\hline 16 & & & $W_{3,3}$ & $W_{3,2}$ & $W_{3,1}$ & $D_{3}$ & & & $W_{2,3}$ & $W_{2,2}$ & $W_{2,1}$ & $D_{2}$ & \\
\hline 17 & & & & $W_{3,3}$ & $W_{3,2}$ & $W_{3,1}$ & $D_{3}$ & & & $W_{2,3}$ & $W_{2,2}$ & $W_{2,1}$ & $D_{2}$ \\
\hline 18 & & & & & $W_{3,3}$ & $W_{3,2}$ & $W_{3,1}$ & $D_{3}$ & & & $W_{2,3}$ & $W_{2,2}$ & $W_{2,1}$ \\
\hline
\end{tabular}

time for the detection of 1 st product droplet. At the end of $1 \mathrm{st}$ macro-cycle, the mixture of the 1st product droplet and substrate droplet stays on Electrode 8 of ME-1, while the 1st washing droplet stays on the leftmost electrode of reactor-1. The spacing between the above two droplets is one meta-electrode. Therefore, they can be synchronously moved along the transport bus by serially activating the electrodes in one meta-electrode.

At the beginning of 2nd macro-cycle, the mixture droplet and 1st wash droplet start moving synchronously along the transport bus with a spacing of one meta-electrode, while the dispensing of the 2nd wash droplet starts from the wash reservoir. The dispensing is synchronized with the transportation. At the end of 2nd macro-cycle, the mixture droplet and the 1st wash droplet reach Electrode 8 of ME-2 and ME1 , respectively; the 2 nd washing droplet stays at the leftmost electrode of reactor- 1 .

During the 3rd macro-cycle, the mixture droplet, and the 1 st and 2nd wash droplets move along the transport bus with a spacing of one meta-electrode, while the $3 \mathrm{rd}$ wash droplet is dispensed from the wash reservoir. At the end of the 3rd macro-cycle, the mixture droplet reaches Electrode 8 of ME-3, and it is followed by three wash droplets. The spacing between two adjacent droplets is one meta-electrode.

From the beginning of the 4th macro-cycle, these four droplets move along the transport bus with a spacing of one meta-electrode, until the mixture droplet reaches the detector at the end of 6th macrocycle. The optical signal from the mixture droplet is detected by the optical detector for five clock cycles, during which the three wash droplets wait a distance of one meta-electrode. After the detection is completed, these four droplets are moved to the waste reservoir.

During the detection of the 1 st product droplet, the 2 nd product droplet is moved from reactor-2 to the leftmost electrode of reactor1. This move operation can be synchronized with the detection of 1st product droplet since there is no conflict in the activation of the pins. Other product droplets, i.e., 3rd, 4th, ..,n $n$th droplets, will also be moved in the same manner as the 2 nd product droplet since the electrodes in the same column of the reactors are controlled by the same input pin. When the 1 st product droplet leaves the detector, the 2 nd product droplet waiting on the leftmost electrode of reactor- 1 starts to traverse the transport bus in the same manner as its predecessor, as shown in Fig. 3. During the detection of the 2 nd product droplet, other product droplets are moved back to their original reactors in order to keep the sample droplets in their respective reactor lanes. Under the pin constraints of the prototype chip layout, $n$ product droplets of $n$ assays can be detected serially with minimum completion time using the above optimal schedule.

The optimal droplet-routing schedule for the 3-plex assay is shown in Table I. We list the operations involving the substrate reservoir, the wash reservoir and the meta-electrodes during each macro-cycle. Let $i$ be the index of the product droplets from 3 different assays. The parameter $D_{i}(i=1,2,3$ and so on but in this example, we limit it to only a 3-plex assay) denotes that the $i$ th product droplet traverses one meta-electrode. The parameter $S B_{i}(i=1,2,3)$ indicates the dispensing of a substrate droplet to mix with the $i$ th product droplet. Finally, $W_{i, j}=1(1 \leq i, j \leq 3)$ if the $j$ th wash droplet for the $i$ th product droplet traverses one meta-electrode. For example, during the 1st macro-cycle, the 1st product droplet $\left(D_{1}\right)$ traverses ME-1, the 1st wash droplet $\left(W_{1,1}\right)$ is dispensed from the wash reservoir, and one substrate droplet $\left(S B_{1}\right)$ is dispensed from the substrate reservoir, as illustrated in Fig. 2. By the end of the 18th macro-cycle, three product droplets of a 3-plex assay have been detected.

Given the prototype chip layout and the pin assignment shown in Fig. 1, the proposed schedule minimizes the completion time of the detection stage for $n$ product droplets. We use the principle of mathematical induction to prove this claim.

First, we consider the base case, i.e., $n=1$ (the detection of 1st product droplet). According to the detection procedure presented above, the movement of the product droplet along the transport bus is synchronized with the mixing of one substrate droplet and the dispensing of three washing droplets. There is no idle time during this process. Therefore, for the prototype chip layout and the pin assignment, the proposed schedule minimizes the completion time for the detection of the 1st product droplet. We next make the induction hypothesis for $n=k$ (the detection of $k$ product droplets). We assume that the proposed schedule yields minimum completion time for the detection of $k$ product droplets. We next consider the induction step for $n=k+1$, i.e., the detection of $(k+1)$ product droplets. From the $n=k$ case, we can see that the completion time for the detection of the previous $k$ product droplets has been minimized. The $(k+1)$ th product droplet waiting at the leftmost electrode of reactor- 1 starts to traverse the transport bus at the same time as the $k$ th product droplet leaves the detector site, which is a requirement for optimality. Therefore, the time interval between the detection of the $k$ th and the $(k+1)$ th product droplets is minimized. From the $n=1$ case, we can see that the completion time for the detection of $(k+1)$ th product droplet is also minimized. Therefore, the completion time for the detection of $(k+1)$ product droplets is minimized. 


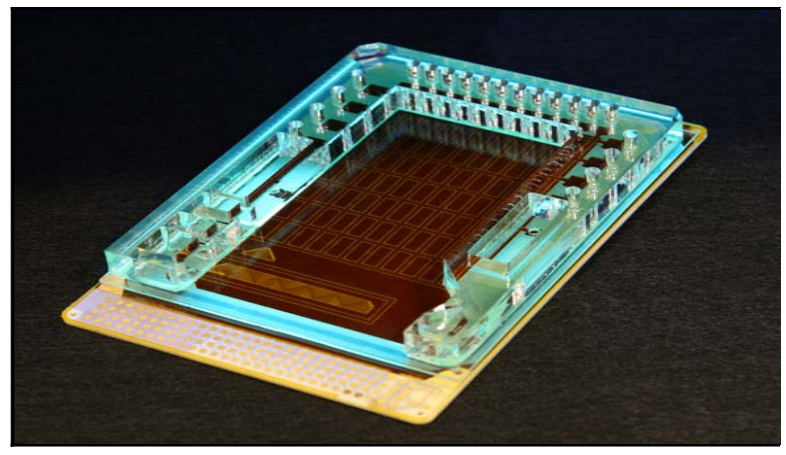

Fig. 4. Fabricated digital microfluidic lab-on-chip used for the $n$-plex assay experiment.

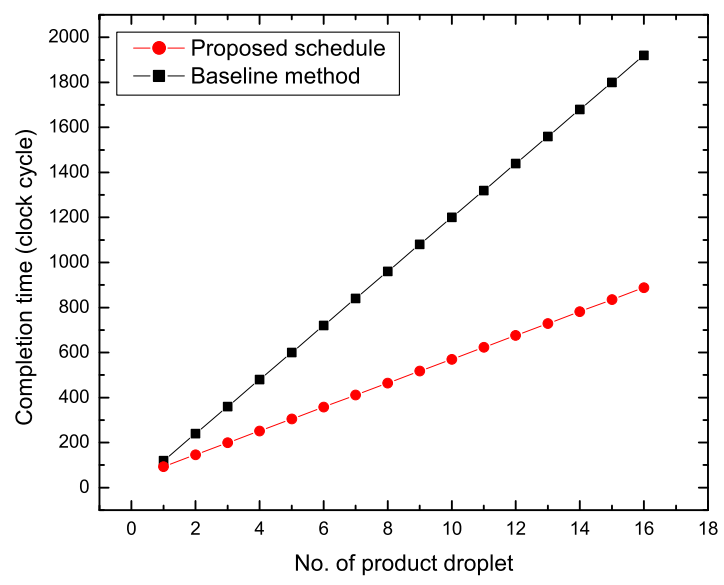

Fig. 5. Comparison of the completion time of the proposed schedule and the baseline schedule for the detection stage of the $n$-plex assay.

\section{EXPERIMENT AND ANALYSIS}

As a typical example of an $n$-plex assay, we perform a 3-plex assay for the diagnosis of acute myocardial infarction (AMI) [3] on a fabricated digital microfluidic platform; see Fig. 4. The fabricated platform consists of 1140 electrodes and 64 input pins. The platform also includes 26 reservoirs for high-throughput processing, with 8 reservoirs storing source reagent solutions, 12 reservoirs storing sample solutions, 2 reservoirs storing the solutions for washing, 1 reservoir storing the substrate solutions, and 3 reservoirs used for waste handling. There are 12 reactors where the reagent and sample droplets are mixed and incubated. The PMT is located at the bottom of the transport bus in the left part of the fabricated platform.

For the 3-plex assay, an example of a human physiological fluid, i.e., serum, is sampled and dispensed to the digital microfluidic labon-chip. Three assays, namely troponin-I, myoglobin, and creatine kinase-MB (CK-MB) measurements, are performed on the physiological fluid. The three product droplets of the 3-plex assay occupy reactor- 1 , reactor- 2 , and reactor- 3 for the detection. Fluidic operations of the 3-plex bioassay are implemented by activating the control pins according to the pin-actuation sequences. The detection stage is implemented using the proposed optimal droplet-routing schedule in Table I. Following the schedule, operations in the detection stage are synchronized, i.e., executed concurrently without any pin-actuation conflict.

Following the proposed schedule, we estimate the duration for the detection stage of the $n$-plex assay. Moving the $i$ th product droplet $D_{i}(i=1,2, \ldots, n)$ from the leftmost electrode of reactor- 1 to the detector located on the Electrode 8 of ME-6 takes 6 macro-cycles.
Since 1 macro-cycle is equal to 8 clock cycles, the above moving process takes $6 \times 8$ clock cycles, i.e., 48 clock cycles. The detection process for each product droplet takes 5 clock cycles. When the last product droplet, i.e., the $n$th droplet is detected, it has to be moved to the waste reservoir. This operation takes $5 \times 8$ clock cycles. In general, the detection stage of the $n$-plex assay takes $(6 \times 8 \times n)+$ $(5 \times n)+(5 \times 8)$ clock cycles, i.e., $53 \times n+40$ clock cycles.

We next compare the proposed method to an unoptimized baseline schedule. In the baseline schedule, the next product droplet that needs to be detected does not move in the transport bus until its predecessor product droplet and the following three washing droplets reach the waste reservoir. In the baseline schedule, the detection stage of the $n$-plex assay takes $[(6+7+8)+(7+8)+(7+8)+8 \times 8] \times n+$ $(5 \times n)$ clock cycles, i.e., $120 \times n$ clock cycles. A comparison of the completion time of the proposed schedule and the baseline schedule for the detection stage of the $n$-plex assay is shown in Fig. 5. For a 3 -plex assay, the baseline method takes 360 clock cycles, while the proposed schedule only takes 199 clock cycles (45\% reduction).

\section{CONCLUSIONS}

We have presented an optimal droplet-routing schedule for the detection stage of the $n$-plex bioassay for a prototype chip layout and the pin assignment. Electrowetting-on-dielectric has been used to implement the digital microfluidic lab-on-chip. We have formally proven that the schedule minimizes the completion time. An on-chip experiment has also been performed to demonstrate the effectiveness of the proposed schedule for a 3-plex assay. The proposed schedule leads to lower completion time than an unoptimized baseline schedule for the detection stage of an $n$-plex assay. The reduction is more striking for larger volumes of $n$.

\section{REFERENCES}

[1] R. B. Fair et al., "Chemical and biological applications of digitalmicrofluidic devices", IEEE Design \& Test of Computers, vol. 24, pp. 1024, 2007.

[2] K. Chakrabarty and F. Su, Digital Microfluidic Biochips: Synthesis, Testing, and Reconfiguration Techniques, CRC Press, Boca Raton, FL, 2006.

[3] A. Chiu et al., "Troponin-I, myoglobin, and mass concentration of creatine kinase-MB in acute myocardial infarction", Oxford $J$ Med, vol. 92, pp. 711-718, 1999.

[4] I. B.-Nad, H. Yang, P. S. Park and A. R. Wheeler, "Digital microfluidics for cell-based assays", Lab on a Chip, vol. 8, pp. 519-526, 2008.

[5] S. O. Flyckt and C. Marmonier, Photomultiplier Tubes: Principles and Applications, Philips Photonics, Brive, France, 2002.

[6] P. Paik et al., "Programmable flow-through real-time PCR using digital microfluidics", MicroTAS, 2007.

[7] V. Srinivasan et al., "Clinical diagnositics on human whole blood, plasma, serum, urine, saliva, sweat, and tears on a digital microfluidic platform", Proc. MicroTAS, pp. 1287-1290, 2003.

[8] V. Srinivasan et al., "An integrated digital microfluidic lab-on-a-chip for clinical diagnostics on human physiological fluids", Lab on a Chip, pp. 310-315, 2004.

[9] R. Sista et al., "Development of a digital microfluidic lab-on-a-chip for automated immunoassay with magnetically responsive beads", Proc. AIChE Annual Meeting, 2007.

[10] S. Fei and K. Chakrabarty, "Architectural-level synthesis of digital microfluidics-based biochips", Proc. IEEE/ACM ICCAD, pp. 223228, 2004.

[11] P.-H. Yuh et al., "A progressive-ILP based routing algorithm for crossreferencing biochips", Proc. DAC, pp. 284-289, 2008.

[12] K. F. Bohringer, "Modeling and controlling parallel tasks in dropletBased microfluidic systems", IEEE Trans. CAD, vol. 25, pp. 334344, 2006.

[13] S.-K. Fan et al., "Manipulation of multiple droplets on $\mathrm{N} \times \mathrm{M}$ grid by cross-reference EWOD driving scheme and pressure-contact packaging", Proc. Int. Conf. MEMS, pp. 694-697, 2003.

[14] T. Xu et al., "Automated design of pin-constrained digital microfluidic biochips under droplet-interference constraints", ACM Journal on Emerging Technologies in Computing Systems, vol. 3, article 14, 2007. 